\title{
A Study of clinical and laboratory profile of children present- ing with febrile seizures in a teaching hospital
}

\section{P Venkateshwar}

\author{
Assistant Professor, Department of Pediatrics, Prathima Institute of Medical Sciences, Nagunur, Karimnagar, Telangana, India \\ Address for communication: Dr. P Venkateshwar, H. No 4-3-4/A Kaman Road Peddapalli- 505172, \\ Email: govu941@gmail.com
}

\begin{abstract}
Background: Febrile seizures are the most common cause of convulsions in children. Although most febrile seizures are benign, the child must be evaluated immediately to reduce parental anxiety, to identify the cause of fever and preventable risk factors if any. It is essential to exclude underlying pyogenic meningitis, either clinically or if any doubt remains, by lumbar puncture. The present study evaluated the common risk factors associated with febrile seizures and the conditions causing fever commonly associated with febrile seizures, the morbidity profile and laboratory profile in children presenting with febrile seizures to identify preventable risk factors if any.
\end{abstract}

Methods : $n=50$ Children in the age group of 6 months to 5 years admitted to the Department of Pediatrics, Prathima Institute of Medical Sciences, Nagunoor, Karimnagar with Febrile Seizures diagnosed as per AAP (American Academy of Pediatrics) guidelines, taken up for the study and compared with 50 other children in the same group with fever but without seizures. Both the study group and control were examined, investigated and compared against each other concerning the clinical and laboratory profile.

\section{RESULTS:}

In the present observational prospective study, the incidence of febrile seizures was more in children less than 24 months of age $(78 \%)$, with a Male children Preponderance (58\%). There were more children with Pallor (70\%), Family History of Seizure Disorder (30\%), and Consanguinity (38\%) in children with Febrile Seizures. URI \& Viral fever (44\%) were the most common etiological factors for fever among the study group. On investigation, many children with Febrile Seizures had low Haemoglobin levels (60\%) and Microcytic Hypochromic

blood picture (58\%). There was no significant abnormality in Total \& Differential Leucocyte Count, Serum Calcium, Electrolyte, Random Blood Sugar levels among the Cases and Controls.

Conclusion: Young age ( $<24$ months), family history of febrile seizures and family history of epilepsy are risk factors for febrile seizures. Consanguinity among parents was an incidental finding in children with febrile seizures. Many children with febrile seizures had Microcytic Hypochromic anemia pointing towards iron deficiency thus iron supplementation to prevent anemia may lead to decreased incidence of development and recurrence of febrile seizures. Association of serious infectious diseases with febrile seizures is rare. Laboratory investigations should be directed towards the identification of the cause of fever and not an evaluation of seizure.

Keywords: Febrile seizures, Risk Factors.

\section{Introduction}

Febrile seizures are one the most common disorder seen during childhood it is usually benign in nature and has a very good prognosis. History of recording of seizures is very old the one by Hippocrates shows a note of febrile seizures when he mentions that convulsions were associated with fever in children less than seven years ago is a common problem which usually is not seen in older children and adults and the parents need not be apprehensive of their child getting epilepsy at a later age in life. ${ }^{[1,2]}$ The overall prevalence of febrile seizures in children is estimated at approximately 3-4\%. ${ }^{[3]}$ Clinically distinction has to be made between those children with febrile seizures and children having epilepsy to ally the apprehensions of the parents as febrile seizures are being in nature. [4] In children with febrile convulsions the risk of long term effects on behavior or intelligence is negligible and the risk of later development of epilepsy is also very low. ${ }^{[4]}$ To identify a child having febrile convulsions the patient has to fulfill AAP criteria which include the child should have febrile illness or certainly fever, neurologically healthy between 6 months and 5 years of age whose seizure is brief ( $<15$ minutes), generalized and occurs only once (simple febrile seizure) or more times (complex febrile seizures) during 24 hours during a fever. Children whose seizures are attributable to a central nervous system infection and those who have had a previous afebrile seizure or central nervous system abnormality are not febrile seizures. Earlier studies have shown the occurrence of certain risk factors for the development of febrile seizures and its recurrence. Earlier studies have shown the occurrence of certain risk factors for the development of febrile seizures and its recurrence. They are young age at onset ( $<15$ months) and epilepsy in first degree relatives, many previous episodes of febrile seizures and first complex febrile seizures. ${ }^{[5]}$ The present study evaluated the clinical aspects, risk factors for development and recurrence, morbidity profile and laboratory profile among the children with febrile seizures in comparison with children having fever but no seizures to identify the etiological factors in patients visiting our tertiary care hospital. 


\section{Material and Methods}

This prospective study was done in the Department of Pediatrics, Prathima Institute of Medical Sciences, Naganoor, Karimnagar. Institutional Ethical committee permission was obtained for the study. Written consent was obtained from the parents/guardians of the children in the study. $N=30$ children aged from 6 months to 5 years who were diagnosed with febrile seizures as per the American Academy of pediatrics criteria admitted to our Tertiary care teaching hospital were studied. The laboratory investigations, risk factors, and morbidity were analyzed during the study. The data were also compared with the $n=30$ children of similar age group with fever but without seizures. Excluded patients were children diagnosed with intracranial infections based on CSF analysis, children with seizures without fever, and children with underlying neurological diseases. All the admitted patients were assessed with a detailed history and a thorough clinical examination was done to find out the cause of fever. Laboratory investigations were done concerning complete blood count, peripheral blood smears, serum RBS, calcium, electrolytes, blood culture and sensitivity, routine urine and microscopy culture and sensitivity (if required) CSF analysis in cases of suspected intracranial infections. All the collected data entered in MS Excel spreadsheet and analyzed by using SPSS version 19 on windows format.

\section{Results}

Among the total cases, $n=30$ children examined in the study $n=10(33.33 \%)$ were between the age group $6-12$ months, $n=12(40 \%)$ of them were in the age group $13-24$ months. Most of the children in this study were below the age group of below 24 months. Among the $n=30$ cases $n=19(63.33 \%)$ were male and $n=11(36.67 \%)$ were females. In the controls, the total number of males were $n=17(56.67 \%)$ and females were $n=13(43.33 \%$ ) shown in (table 1 )

Table 1: Showing the percentage of cases along with age distribution

\begin{tabular}{|l|c|c|c|c|}
\hline $\begin{array}{c}\text { Age in } \\
\text { months }\end{array}$ & $\begin{array}{c}\text { Number of } \\
\text { cases }\end{array}$ & Percentage & $\begin{array}{c}\text { No. of } \\
\text { patients }\end{array}$ & Percentage \\
\hline $06-12$ & 10 & 33.33 & 11 & 36.67 \\
\hline $13-24$ & 12 & 40.00 & 09 & 30 \\
\hline $25-36$ & 05 & 16.67 & 07 & 23.33 \\
\hline $37-48$ & 02 & 6.67 & 02 & 6.67 \\
\hline $49-60$ & 01 & 3.33 & 01 & 3.33 \\
\hline Total & 30 & 100 & 30 & 100 \\
\hline
\end{tabular}

The percentage of cases with consanguinity among the $n=30$ cases studied showed $n=10(33.33 \%)$ had second-degree consanguinity and $n=5(16.67 \%)$ had 3rd-degree consanguinity and remaining $n=16(53.33 \%)$ were non-consanguineous. In the control patients, the non-consanguineous marriages were in $n=23(76.67 \%)$ of the parents.

Chart 2: The number of consanguinity of marriages of cases with controls

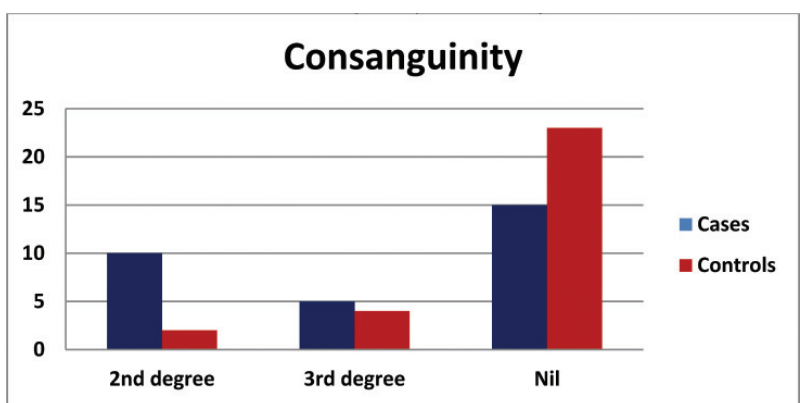

The distribution of family history of febrile seizures among the cases and controls revealed that there were $n=10(33.33 \%)$ of cases in the study group are with a family history of febrile seizures. There was no family history of febrile seizures in the control group. The $p$ values were $<0.05$ which were found to be significant. The family history of Epileptic seizure disorders revealed that in cases $n=3(10 \%)$ had a family history of seizure disorders and no family history of seizure disorder was found in the control group.

Table 2: showing the distribution of family history of febrile seizures among cases and controls

\begin{tabular}{|c|c|c|c|c|}
\hline $\begin{array}{c}\text { Family History of } \\
\text { Febrile Seizures }\end{array}$ & Cases & Percentage & Controls & Percentage \\
\hline No & 20 & 66.67 & 30 & 100 \\
\hline Yes & 10 & 33.33 & 00 & 000 \\
\hline Total & 30 & 100 & 50 & 100 \\
\hline
\end{tabular}

The physical examination of the patients revealed that the number of children with pallor in the study group was $\mathrm{n}=21(70 \%)$ as compared to the controls were pallor was found in $n=9(30 \%)$ of the children. The $p$ values were found to be less than $<0.05$ which is significant. Similarly, the systemic examination revealed no tachypnea was found in a study group whereas tachypnea was found in $n=2(6.67 \%)$ and $n=25(83.33 \%)$ of the patients of the control group $p$ values were $<0.02$ hence considered significant (table 3).

Table 3: The General physical examination and systemic examination

\begin{tabular}{|l|c|c|c|}
\hline & Cases & Controls & P-value \\
\hline Pallor & \multicolumn{2}{|l|}{} \\
\hline Present & 21 & 09 & \multirow{2}{*}{$<0.05^{*}$} \\
\cline { 1 - 3 } Absent & 09 & 21 & \\
\hline
\end{tabular}




\begin{tabular}{|l|l|l|l|}
\hline \multicolumn{4}{|l|}{ Systemic Examination } \\
\cline { 1 - 3 } Normal & 28 & 05 & \multirow{2}{*}{$<0.02 *$} \\
\hline Tachypnea & 02 & 25 & \\
\hline
\end{tabular}

* Significant

The distribution of etiological factors in this study found $\mathrm{n}=12(40 \%)$ children were with viral and URI fevers in the study group. The number of patients with URI in the controls was $\mathrm{n}=15(50 \%)$ and viral fevers in $\mathrm{n}=10(33.33 \%)$ and LRI in $n=08(26.67 \%)$ patients of the control group details shown in table 4.

Table 4: Distribution of etiological factors of fever among cases and controls

\begin{tabular}{|l|c|c|c|c|}
\hline $\begin{array}{c}\text { Etiology of } \\
\text { fever }\end{array}$ & Cases & Percentage & Controls & Total (\%) \\
\hline URI & 12 & 40.00 & 15 & 50.00 \\
\hline Viral Fever & 10 & 33.33 & 08 & 26.67 \\
\hline LRI & 0 & 0.00 & 3 & 10.00 \\
\hline Diarrhea & 3 & 10.00 & 1 & 3.33 \\
\hline Enteric Fever & 2 & 6.67 & 2 & 6.67 \\
\hline Dysentery & 2 & 6.67 & 1 & 3.33 \\
\hline UTI & 1 & 3.33 & 0 & 0.00 \\
\hline Total & 30 & 100 & 30 & 100 \\
\hline
\end{tabular}

The laboratory investigations in the study showed that low hemoglobin levels were found in $n=18(60 \%)$ children of the study group and $n=9(30 \%)$ children in the control group. The $p$ values were less than 0.05 which is statistically significant. The type of anemia in the peripheral blood smear was mostly microcytic hypochromic. The Total Leucocyte Counts in the study group were found within the normal ranges and one child in the study group has raised leucocytic count and neutrophilia. The serum electrolytes, calcium, and RBS in both groups were found to be in the normal range.

\section{Discussion}

In the present study, we compared the risks for febrile convulsions with controls with fever but without convulsions. The majority of cases of febrile convulsions were seen below 2 years of age. It has been attributed to the difference in water and electrolyte balance, increased oxygen consumption and diminished dendritic connections and electrophysiologic differences between the adult brains these are some of the possible reasons for convulsions in this age group. It has also been hypothesized that the rapid rates of cerebral maturation and myelination at two years have shown decreased cases of febrile convulsions after 2 years. ${ }^{\left[{ }^{6]}\right.}$ It was found in this study that children of a lower age group than 2 years also more susceptible to infections and fevers. It has been associated with the waning of passive immunity by 6 months of age and exposure to community-acquired infections. Similar studies in this field have shown that febrile convulsions are common under 2 years of age agreeing with the results of the present study. ${ }^{[7-10]}$ The risk factor analysis has shown that family history of febrile seizures in $n=10$ (33.33\%) of children out of which $23.33 \%$ had a family history of febrile convulsions and $n=3(10 \%)$ had a family history of epilepsy. In the control group, no history of seizures was found. Nelson KB et al; ${ }^{[7]}$ have shown that children with febrile convulsions who have a positive family history of febrile seizures were associated with 3 fold increase in the rate of epilepsy later in life compared with those without a history of seizures. Frantzen et al; have shown that the incidence of family history of epilepsy in $20 \%$ of cases and $40 \%$ in children with febrile seizures. ${ }^{[11]}$ Studies by NCPP showed that positive family history for seizure disorder makes an important contribution to the risk of febrile convulsions and that febrile convulsion in the mother has the greatest influence. ${ }^{[7,12]}$ The association of consanguinity among the parents of children with febrile seizures, mainly second degree as compared to the control group which comprised of children without seizures showed the role of genetics in the development of febrile seizures. The mode of genetic inheritance has been attributed to polygenic mechanism but the definitive mode of inheritance is still not clear. ${ }^{[13-15]}$ In the present study a significant number of children $60 \%$ in the study group were found with pallor and low hemoglobin levels and microcytic hypochromic blood pictures indicating Iron deficiency anemia. L Kumara et al; in a similar study has shown that iron deficiency anemia is a significant risk for simple febrile seizures in children from 6 months to 3 years. ${ }^{[16]}$ Azhar et al; in their study round the relation of the iron store and first febrile seizure by a prospective case-control study. They concluded that plasma ferritin levels are significantly lower in children with first febrile seizures suggesting the role of IDA. [17] Therefore it appears that a complex interaction among the immune-inflammatory process, cytokine activation, and genetic factors are involved in the pathogenesis of febrile seizures. Most of the febrile seizures are self-limiting and low risk of morbidity and mortality and are relatively benign however long term management requires a thorough assessment and risk stratification to devise a customized plan for each child.

\section{Conclusion}

Within the limitations of the present study, it can be concluded that febrile seizures are more common in children below 24 months of age and mostly occurs in male children. History of consanguinity was present in many cases. The laboratory profile showed children with febrile seizures having low 
hemoglobin levels and suffering from IDA. Therefore treating physicians must be aware of risk factors and address them appropriately during handling such cases.

\section{REFERENCES}

1. Ouellette EM. The child who convulses with fever. Ped Clin N Am 1974;21:467-81.

2. Andrew Sparman. Convulsions in Von Resentin. The Diseases of children and their remedies 10th edition London. Classics of Medicine 2004;345.

3. Abolfazl Mahyar, Parviz Ayazi, Mazdak Fallahi, Amir Javadi. Risk factors of the febrile seizures in Iranian children.Int J Pediatr.2010June:24-26.

4. Verity Christopher M. Do Seizures Damage Brain? The Epidemiological Evidence. Arch Dis Child1998; 78:78-84.

5. William $\mathrm{H}$ Trescker, Renald P Lescher. The Epilepsies. In Walter G Bradley, Robert B and Gerald M. Neurology in Clinical Practice4ndEd.Philadelphia. Butterworth Heinemann 2004; 2:1953-93.

6. Rutter Nand Smales O.R.C. Role of Routine Investigations in Children presenting with their First Febrile Convulsion. Arch Dis Child 1977; 52:188-191.

7. Nelson, Karin B, Ellenberg, Jones H. Prognosis in Children with Febrile Seizures. Pediatrics.1978; 61:720-727.

8. Leung AK, Robson WL. Febrile convulsions. How dangerous they are? Postgrad Med 1991; 89(5):271-222.

9. Shah Samir, Alpern Elizabeth R, Zwerling Lisa Z, Jennifer $R$, Karin L, Louis $M$. Low risk of bacteremia in children with febrile seizures. Arch Pediatr Adolesc Med.2002; 156:469-472.

10. Berg AT, Berkovic SF, Brodie MJ, Bchhalter J, Cross JH, Van Emde Boas W, et al; Revised terminology and concepts for organization of seizures and epilepsies. Report of ILAE commission on classification and terminology 2005-2009. Epilepsia 2010; 51(4)676-85.

11. Frantzen E, Lennox-Buchthal MA, Nygaard A. Longitudinal EEG and Clinical Study of Children with Febrile Convulsions. Electroencephalogr Clin Neurophysiol 1968;24:197.

12. Nelson, Karin Band Deborah G Hirtz. Febrile Seizures. In Swaiman Kenneth F, Pediatric Neurology-Principles and practice, 1stEd. Toronto. The C.V.MosbyCo.1989; 439-442.

13. Talukdar B. Febrile Seizures. In Takudar B. The Essentials of Pediatric Neurology1stEd.New Delhi. New Age International (P) Ltd.1997;175-182.

14. Hauser WA, Annegers JF, Anderson VE, Kurland LT. The Risk of Seizure Disorders among Relatives of Children with Febrile convulsions. Neurology1985; 35:1268-73.
15. Brett Edward. Epilepsy and Convulsions. In Brett EM Ed. Pediatric Neurology 3rd Ed. Edinburgh. Churchill Livingstone.1997;20:270-282.

16. Leela Kumari P, MKC Nair, SM Nair, Lalitha Kailas, and S Geetha. Iron deficiency as a risk factor for simple febrile seizures-A case-control study. Indian Pediatr 2012; 49:1719.

17. Daoud AS, Batieha A, Abu-Ekteish F, Gharaibeh N, Ajlouni S, Hijazi S. Iron status: Possible Risk Factor for the First Febrile Seizure. Epilepsia.2002; 43:740-33.

How to cite this article : Venkateshwar P. A Study of clinical and laboratory profile of children presenting with febrile seizures in a teaching hospital. Perspectives in Medical Research 2020; 8(1):55-58

Sources of Support: Nil, Conflict of interest: None declared 